in $64 \%$. Adverse effects including brain loss were severe in $7 \%$, moderate in $23 \%$, and slight in $47 \%$. Low-dose synthetic ACTH therapy was as effective in WS as higher doses used in previous studies. (Ito M, Aiba H, Hashimoto $\mathrm{K}$ et al. Low-dose ACTH therapy for West syndrome. Initial effects and long-term outcome. Neurology January (1 of 2) 2002;58:110-114). (Reprints: Dr Masatoshi Ito, Department of Pediatrics, Shiga Medical Center for Children, 5-7-30 Moriyama, Moriyama 524-0022, Japan).

COMMENT. Low-dose synthetic ACTH therapy for West syndrome is effective and is associated with less toxicity than higher dose regimens. Control of seizures and EEG hypsarrhythmia and favorable long-term outcome are correlated with a cryptogenic etiology and avoidance of delay in initiation of ACTH treatment. The frequency of serious adverse effects may be decreased by using the lowest effective dose of ACTH for the shortest duration. Ito and his colleagues have previously reported on the effectiveness of low-dose ACTH regimens for infantile spasms (Pediatr Neurol, 1990; see Progress in Pediatric Neurology I, PNB Publishers, 1991;pp 33-34). For a $10 \mathrm{~kg}$-10 mos old infant, the dose of ACTH used in Japan was approximately 10 units daily and 300 units total. While these doses are similar to those advocated from my own experience (IAMA, 1962) and doses currently employed by my colleagues (Drs Nordli, Stack, \& Swisher) at Children's Memorial Hospital, Chicago, much larger amounts were favored by Snead in the US (1989) and by my colleagues (Drs Wilson \& Brett, 1987) at the Hospital for Sick Children, Great Ormond Street, London. From a practical standpoint, the use of ACTH in the natural form in the US has become difficult because of short supply and price constraints (approx $\$ 1000$ per vial). The synthetic form is thought to be more potent and toxic than the natural form, but controlled studies are lacking. In conclusion, ACTH can be an effective and relatively safe treatment for infantile spasms, when injections are initiated soon after diagnosis, doses are conservative, and the lowest effective amount is used for the shortest time. Toxicity may be minimized by close and frequent patient monitoring.

A symposium on West syndrome, edited by Fukuyama Y, Tokyo, Japan ( Dev 2001;23:624-648), included several papers on treatment. Hancock E et al, from the UK, in reviewing the literature on the effectiveness of vigabatrin compared to ACTH and predisone, concluded that the optimum therapy for West syndrome remains uncertain. Ito, $M$, reporting on practice in Japan, comments that nonhormonal treatments are tried before using ACTH. He adds that close monitoring of adverse effects, especially subdural hematoma, is important, even with low dose synthetic ACTH therapy. Riikonen R of Finland recommends low-dose ACTH as the first choice of treatment and preferable to vigabatrin, since side effects are well known, treatable, and reversible. Baram $\mathrm{TZ}$ of Irvine, CA, reviewing the reasons for differences regarding ACTH dosage among researchers, emphasizes the variability in ACTH preparations, their bio-availability, and possible genetic and environmental differences among patients that determine responsiveness to ACTH.

\title{
VIGABATRIN ASSOCIATED RETINAL PATHOLOGY
}

A prospective study of 29 children treated with vigabatrin (VGB) as add on therapy for epilepsy included ophthalmic examination before and at 6-month intervals for 6.5 years, at Sultan Qaboos University Hospital, Sultanate of Oman. Age of onset of seizures ranged from birth to 5 years (mean, 13 months). Of 21 fulfilling study requirements and follow-up, 6 had West syndrome, 3 had Lennox Gastaut syndrome, and the remainder, partial or mixed seizures. Eighty percent had psychomotor retardation. Dose of VGB was $25-114 \mathrm{mg} / \mathrm{kg} /$ day (mean $56 \mathrm{mg}$ ) and treatment duration was 6-85 months (mean 36 mos). Four (19\%) taking 25-50 
$\mathrm{mg} / \mathrm{kg} /$ day for 33-81 months developed retinal pigmentation, hypopigmented retinal spots, and optic atrophy. Visual evoked potentials, performed in 21 , were abnormal initially in 2 and became abnormal during VGB therapy in 14. Electroretinography and electro-oculography were not available. (Koul R, Chacko A, Ganesh A, Bulusu S, Al Riyami K. Vigabatrin associated retinal dysfunction in children with epilepsy. Arch Dis Child Dec 2001;85:469-473). (Respond: Dr R Koul, Department of Child Health (Division of Paediatric Neurology), Sultan Qaboos University Hospital, Al Khod, PO Box 38, 123, Sultanate of Oman).

COMMENT. Regular eye examination including perimetry is recommended in patients treated with vigabatrin. Infants with epileptic syndromes and mental retardation, in whom perimetry is not appropriate, should receive funduscopic examination at least every 3 to 6 months, and retinography, electro-oculography, and VER when available. Retinal changes and visual field constriction associated with VGB can be irreversible, and risks may outweigh benefits if alternative less toxic treatments are available. (see Ped Neur Briefs Dec 2001;15:94-95).

\section{X-LINKED MENTAL RETARDATION AND EPILEPSY SYNDROME}

A kindred of 7 affected male infants with an X-linked mental retardation and epilepsy syndrome (XMRE), distinct from X-linked West and other mental retardation-epilepsy syndromes, is reported from the University of Michigan, Ann Arbor, MI. The locus for the new syndrome is identified on chromosome Xp21.1-p11.4. The tetraspanin gene, implicated in nonspecific mental retardation, was not involved. Seizures were primarily generalized, tonic-clonic, and atonic and began at 4 to 14 months of age (average age, 6.8 months). None had infantile spasms or hypsarrhythmia, and other than mental retardation and epilepsy affecting all patients, and mild rigidity and ataxia in 2, the neurologic examination was unremarkable. MRI, EMG, NCS, and laboratory metabolic tests were normal. (Hedera P, Alvarado D, Beydoun A, Fink JK. Novel mental retardation-epilepsy syndrome linked to Xp21.1-p11.4. Ann Neurol January 2002;51:45-50). (Respond: Dr John K Fink, 5214 Cancer Center Geriatrics Center Building, 1500 E Medical Center Drive, Ann Arbor, MI 48109).

COMMENT. X-linked mental retardation is clinically heterogeneous and is reported in $25 \%$ of all MR. The authors cite references to 202 XLMR genetically mapped syndromes and 33 identified XLMR genes. Seizures occur with several XLMR disorders, and West syndrome is sometimes transmitted as an X-linked disorder mapped to a locus on $\mathrm{Xp} 21.3-\mathrm{Xp} 22,1$, and distinct from the present novel family. In patients without recognizable metabolic or developmental brain abnormalities, a search for X-linked genetic factors is important in XMRE syndromes.

\section{AUTOSOMAL DOMINANT EPILEPSY SYNDROME LINKED TO 2p11}

A newly recognized autosomal dominant epilepsy syndrome with linkage to chromosome 2p11.1-q12.2 is described in an Italian pedigree of 8 patients reported from the Neurosciences Unit, Institute of Child Health and Great Ormond Street Hospital for Children, London, UK. Onset between ages 12 and 50 years, the syndrome is characterized by distal, semi-continuous rhythmic cortical myoclonus, generalized tonic-clonic and complex partial epileptic seizures (ADCME). The majority had only occasional seizures. Seizures were intractable in 3 who developed mild mental retardation. Valproate was most effective, while carbamazepine controlled seizures but exacerbated myoclonus. Interictal EEG abnormalities were generalized and focal frontotemporal. EMG studies of the 